\title{
A realization of load balancing based on distributed real-time database isomerism cluster
}

\author{
Jia $\mathrm{Wu}^{1, \mathrm{a}}$, Dan $\mathrm{Su}^{1}$, Huanyuan $\mathrm{Li}^{1}$, Lei1 $\mathrm{Yan}^{1}$, Xianhui $\mathrm{Li}^{2, \mathrm{~b}}$, ShengPeng $\mathrm{Ji}^{2}$, \\ Jingbo $\mathrm{Li}^{3}$ \\ ${ }^{1}$ JiBei Electric Power Corporation, Beijing 100025, China; \\ ${ }^{2}$ China Realtime Database Co.,Ltd , Nanjing 210000, China. \\ aalice.0729@163.com, 'lixianhui@sgepri.sgcc.com.cn
}

Keywords: Load balancing; real-time database; database cluster; intelligent dispatch algorithm

\begin{abstract}
This paper briefly introduced the technical features of real-time database and distributed database. This paper also introduced real-time database platform based on CORBA component technology, and real-time database isomerism cluster which is the core part of real-time database platform. Based on the above techniques, this paper proposed a realization of load balancing. It adopts dynamic feedback mechanism to optimize the communication mechanisms of load information collection, and gives an intelligent allocation method to improve the overall system performance. Using this kind of load balancing method improved both performance and stability of the cluster.
\end{abstract}

\section{Introduction}

Real-time data collected in power business applications is mainly from the dispatch automation systems, energy metering systems, protection and fault management systems, distribution automation systems, weather systems and so on[1]. With the development of intelligent electric grid, power business is being more and more, and the data scale is increasingly large [2]. The demand for breaking information barriers, and unified data management becomes more intense. Business-to-break information barriers, the demand for unified data management and more intense. There is an urgent need to provide a real-time data platform to meet the needs of integration of the growing mass data storage access and the old system. The vast amounts of data required from the old system need to store in the real-time data platform and run on a real-time database cluster in multiple servers. This requires a load-balancing method to ensure the performance of each server has been fully utilized [3].

\section{Real-Time Data Platform}

Real-time database cluster is an important part of real-time data platform [5]. The real-time database described in this article follows the IEC61970 [6] standard, and achieves the summary, interactive and other functions for real-time data. The core of real-time database above is the CIM model [7] and real-time database cluster.

CIM model is an important part of IEC61970 series of standards. It is an abstract model, covering all the major objects of power companies. It is a standard method for power system resources through providing object classes and attributes and relations between them.

Following IEC61970 is the need of the uniform application of the system model management. Access Layer provides CIS interface, Web Service, OPC, ODBC and other data access forms. Figure1 describes the system structure. Data layer includes relational database and real-time database, relational database can take various commercial database. Real-time database can be any vendor offerings. In order to mask the differences between the various real-time databases, it defines a set of "Real-time Database General Access Interface". Due to the need performance, basically all kinds of real-time database provides $\mathrm{C} / \mathrm{C}++$ secondary development interface. Reference the design of API of 
major mainstream real-time database, the cluster system defines standard interfaces including measuring point management and real-time data access and so on [8].

As show in Fig. 2 External Services provided by platform include: cluster service and CIS services (include GDA、HSDA、TSDA、GES). They are encapsulated through CORBA to achieve purposes cross-platform and cross-language.

Platform achieves load balancing in server by real-time database clustering and load balancing. It uses Balance Service of CORBA for network load balancing.

Real-time database cluster constitutes a virtual single database logical image through at least two or more servers, and provides transparent data services to client as a single database system [9].

\section{Real-time database cluster}

The cluster achieves transparent accessing to heterogeneous databases through a proxy service implementation. It has a set of efficient and accurate measuring point synchronous mechanism, security and management mechanisms, load balancing mechanism.

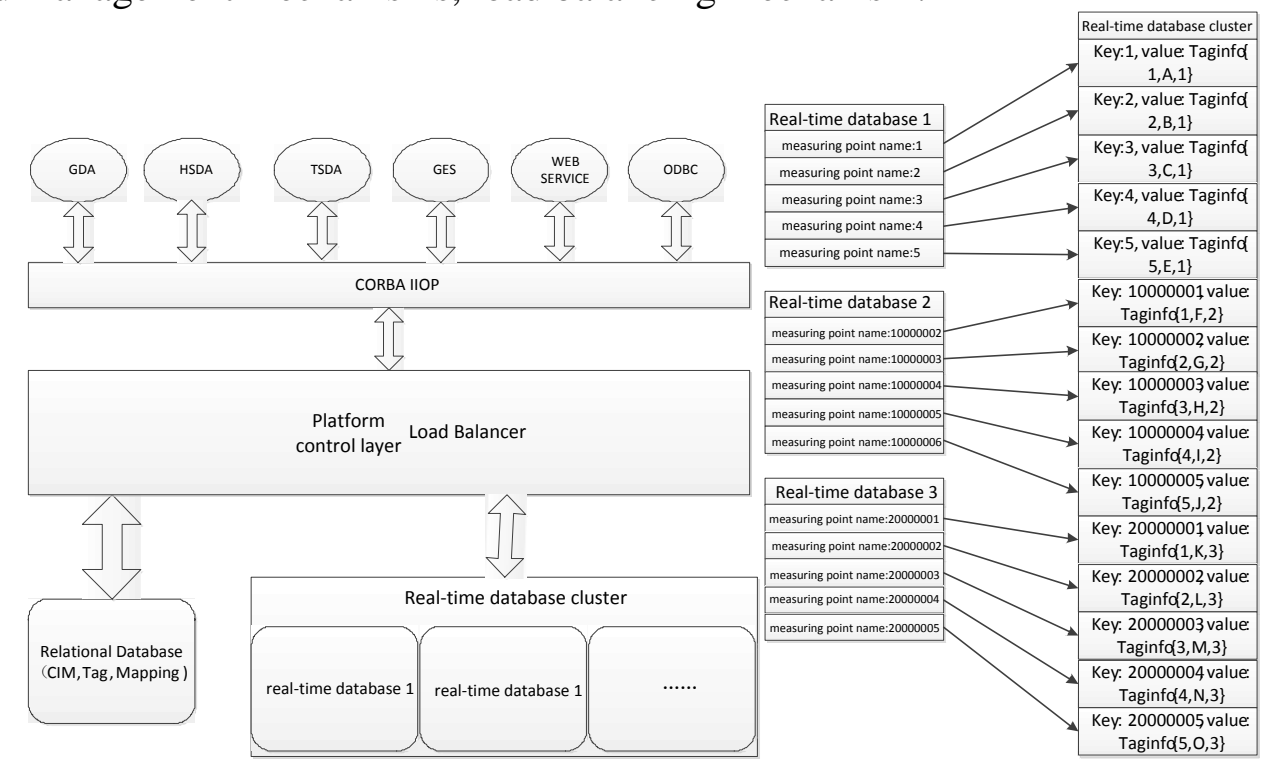

Fig. 1 Platform Architecture Figure Fig.2Synchronization renderings

Universal real-time database access interface: cluster UAPI. Cluster defines a common real-time database access interface, and the client can access the cluster just like accessing a single database system. But it needs to distinguish between different types of databases to dynamic library calls when accessing bottom layer of real-time database, which makes the client become very complicated to achieve the logic. To simplify client underlying logic, it adds heterogeneous real-time database shield between real-time database access interface and cluster common access interface. Its function is equivalent to the agent, the different types of real-time databases work together within the data platform, and the method of external real-time database accessing heterogeneous clusters is the same as real-time database. Agency services inform the type of database through the cluster service when it calls the real-time database interface. And use dynamic invocation dynamic library technology, then Agency services call types of real-time database access interface to access to the database and achieve data CRUD.

Measuring point synchronization mechanism. Cluster internal maintain a structure Taginfo of cluster measuring points, and the structure is correspondence to the HashMap in memory, also Taginfo contains the measuring point ID, description, database ID and other information. All measuring points in database has only one corresponding cluster measuring point structure in cluster. When sync measurement point information, it will loop through each measuring point in database, access the information of point and store in the structure Taginfo. Then store the key-value pair consisting of the measuring point name and Taginfo in HashMap, and if there are repeated measurements names cluster will complain and recorded in the log. Finally, all information of 
measuring points in database will be stored in cluster. The effect of synchronized shows in figure 2, the structure of Taginfo \{measuring points ID, description, belongs database number .

When a client operates on the measuring points such as addition and deletion, then hash Map within a cluster will make the corresponding changes.

When a client queries a measuring point through cluster, the cluster will quickly find the corresponding database and the corresponding measuring point based on the hash keys Map.

Security and Management. In order to monitoring operating status of data platform and determine the access status of the cluster, it sets up a monitoring mechanism and adds service monitoring and control process in the real-time database and the Cluster service machine respectively.

It maintains the number and location information of real-time database through management function. It provides two ways which are based on the remaining number of static load balancing and manually setting, when add new measuring point. The method based on the remaining number of static load balancing is that when metadata management module receives the request to increase measuring point, it selects the maximum percentage of the remaining measuring points to add measuring point based on the percentage of the remaining measuring point in all real-time database cluster in real time. Manually set requires measuring point increase containing the identification information of the specified real-time database.

\section{Load Balance}

Load balance usually consists of multiple servers in a symmetrical manner to form a server cluster and it is controlled by load balancer deploying on a server.

Load balancing usually consists of multiple servers in a symmetrical manner to form a server cluster by deploying on a server load balancer control. Each server has the equivalent status, can provide services outside alone without the aid of other servers. System evenly distributes the external request to one of the server in symmetrical structure through load balancing technology, or shares the heavy load node task on multiple servers to do parallel processing, finally the server receives a request independently respond to customer requests.

Load balance can equally distribute client requests to the server cluster to provide rapid access to critical data for the resolve of concurrent access service issues [10] [11].

Load balancing system framework. Establish LD (load balancer) and RS (clusters) performance synchronous communication mechanism that is the dynamic feedback mechanism in the load balancing system [12], through measuring point including each server performance information stored in real-time database. Load balancer can know the performance information of backend RS through querying measuring points, and when allotting request it priority allocates requests to load low node. For high load RS node, temporarily stop distribution request and at each time period to determine whether the high load node back to normal. Specifically logic shows in Figure3.

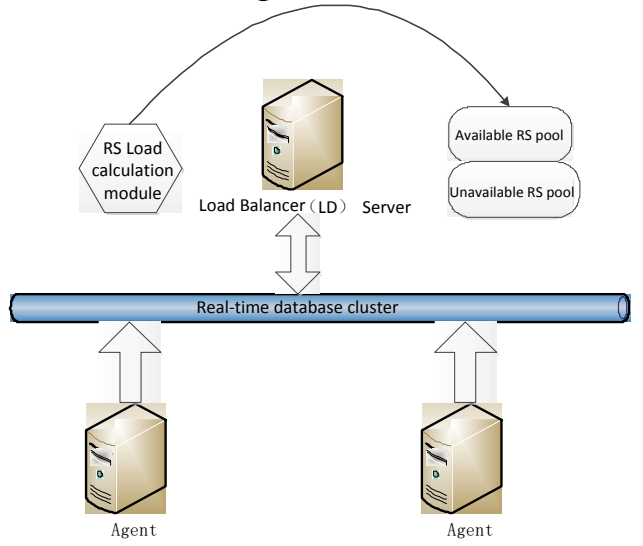

Fig.3 load balancing system framework

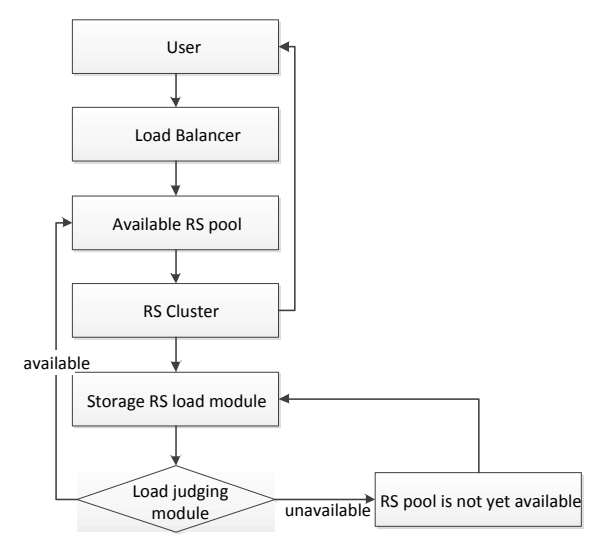

Fig.4 system flow

Intelligent allocation strategy. ntelligent allocation strategy can dynamically monitor the load information for each server, adds nodes flexible and shield unavailable nodes, the specific 
implementation shown in figure 4.

(1) Adding a separate module "monitor" on the load balancer, query data of measuring point corresponding each "agent" through real-time database cluster, judge the health condition of RS;

(2) LD requires additional two shared memory to store the output, store the "available RS" and "unavailable RS". When LD allots request, it needs to read information from "available RS pool" and forwarded the request.

(3) RS processes the request, at the same time "agent" will monitor server node load real time, and write data in measuring point corresponding to real-time database through cluster according to period. Monitor module determines whether RS can continue to process more requests by rules based on the information written.

(4) If the RS load information is normal, put it into the "available RS pool" and wait for the LD allotting request; if the RS load information abnormal, put it into "unavailable RS pool". Meanwhile LD also need to monitor RS in "unavailable RS pool".

\section{Conclusion}

This paper presents a method of real-time database heterogeneous cluster and load balancing. With the development of power informatization, the demand for mass data storage access is more and more urgent due to the rapid growth of the size of the real-time data. The CIM model and GIS components specification described in IEC61970 are the important criterion followed by power software. So real-time database cluster following IEC61970 standard proposed meets the demand. At the same time, the load balance with smart allocation policy combines a real-time database cluster function, it greatly optimizes the performance and stability of the platform for each server, and makes the cluster serve electricity customers better.

\section{References}

[1] Liu Hui. Power Business Quality Management Methodology and Practice [J]. Chinese Electronic Commerce, 2011,10(83):100.

[2] Xiao Shijie . Consideration of Technology for Constructing Chinese Smart Grid [J]. Automation of Electric Power Systems, 2009, 33(9): 1-4.

[3] Yan Lei, Shi Jingxin. On Better Utilization of Idle Resources in Cluster Environment [J]. Science Technology and Engineering,2008,5(8):1173-1176

[4] Kong Jie, Liu Yang. The key technologies and future prospects of Real-time[J].Digital Technology and Application,2014,4:215-216

[5] He Jiang, Wu Xingping, Li Lixi, Fan Tao, Mi Weimin. A COMPONENT BASED REAL-TIME DATABASE MANAGEMENT PLATFORM [J]. Power System Technology,2002,26(3):64-67

[6] IEC61970-1 Energy management system application program interface(EMS-API) [S], Geneva: ISO/IEC ,2005.

[7] Zhou Lin, Wang Nie. Research IEC61970 CIM Model [J]. Science \& technology view, 2013,1:89-89.

[8] Zeng Rong, Yang Shuqian, Jia Yan, Zhang Wen. Design and Implementation of CORBA based Middleware Management [J]. Application Research of Computers,2005,22(2):118-120.

[9] Qu Gang, Deng Jianqing, Han Yunlu. Research on Linux C luster Techno logy [J]. Application Research of Computers, 2005,22(5):100-101.

[10] Tang Jun. The Research and Application of Dynamic Feedback Load Balancing System Based On Cluster [D]. Tianjin : TianjinUniversity, 2008: 11-12.

[11] Zhu Zhixiang, Xu HuiHui, Wang Xiong. Elastic load balancing solution based on cloud computing [J]. Journal of $\mathrm{Xi}^{\prime}$ an University of Posts and Telecommunications, 2013, 18(6): 43-47.

[12] Tian ShaoLiang, Zuo Ming, Wu ShaoWei. Improved dynamic load balancing algorithm 
based on feed_back [J]. Computer Engineering and Design, 2007， 28(3): 572—573 